\title{
Quantum phase transitions of atom-molecule Bose mixtures in a double-well potential
}

\author{
A. Relaño, ${ }^{1}$ J. Dukelsky, ${ }^{2}$ P. Pérez-Fernández, ${ }^{3}$ and J. M. Arias ${ }^{4}$ \\ ${ }^{1}$ Departamento de Física Aplicada I and GISC, Universidad Complutense de Madrid, 28040 Madrid, Spain \\ ${ }^{2}$ Instituto de Estructura de la Materia, CSIC, Serrano 123, 28006 Madrid, Spain \\ ${ }^{3}$ Departamento de Física Aplicada III, Universidad de Sevilla, Sevilla, Spain \\ ${ }^{4}$ Departamento de Física Atómica, Molecular y Nuclear, Universidad de Sevilla and Unidad Asociada to IEM (CSIC) Madrid, Spain
}

(Received 9 June 2014; published 27 October 2014)

\begin{abstract}
The ground state and spectral properties of Bose gases in double-well potentials are studied in two different scenarios: (i) an interacting atomic Bose gas, and (ii) a mixture of an atomic gas interacting with diatomic molecules. A ground state second-order quantum phase transition is observed in both scenarios. For large attractive values of the atom-atom interaction, the ground state is degenerate. For repulsive and small attractive interaction, the ground state is not degenerate and is well approximated by a boson coherent state. Both systems depict an excited state quantum phase transition. In both cases, a critical energy separates a region in which all the energy levels are degenerate in pairs, from another region in which there are no degeneracies. For the atomic system, the critical point displays a singularity in the density of states, whereas this behavior is largely smoothed for the mixed atom-molecule system.
\end{abstract}

DOI: 10.1103/PhysRevE.90.042139

PACS number(s): 05.30.Rt, 05.70.Fh, 64.70.Tg, 03.75.Lm

\section{INTRODUCTION}

Bose-Einstein condensation in dilute atomic gases provides a valuable tool to study fundamental problems of quantum systems at a macroscopic scale. Among such phenomena, the quantum transition from Josephson oscillations to selftrapping is one of the most interesting. It comprises the transition from a regime in which two spatially separated Bose-Einstein condensates (BECs) oscillate, to another one in which the macroscopic wave function remains trapped in one of the two trap sides. This transition has been experimentally observed in [1] by means of a condensate of ${ }^{87} \mathrm{Rb}$ atoms in an optical trap. It has also been theoretically studied in Refs. [2-4]. Using a two-site Bose-Hubbard Hamiltonian as a description of two BECs that can tunnel between the two wells, it has been shown that the quantum phase transition (QPT) develops at a critical value of the atom-atom interaction. The imbalance, that is, the difference of population between the two wells, acts as an order parameter for the transition, changing from zero in the Josephson oscillation regime to a nonzero value in the self-trapping regime.

This is an example of QPT which has been studied in other systems. In [5], the same kind of transition was reported in a nonlinear version of the same system, and it was related to a classical bifurcation in the Hamiltonian. In [6], a similar bifurcation was linked to a transition from zero to nonzero values in the imbalance.

In this work, we expand these studies by focusing not only in the ground state QPT, but also looking at quantum critical phenomena in the energy spectrum. In particular, we will describe the so called excited-state quantum phase transitions (ESQPTs). These occur when some kind of nonanalytic behavior [7] arises in an excited region of the energy spectrum, normally a singularity in the density of states or in the flow of the energy spectrum through the critical energy line. In contrast to usual quantum phase transitions [8], they are not characterized by a critical value of the control parameter $\lambda_{c}$, but by a critical energy $E_{c}$ above the ground state, at which the nonanalyticity takes place. Usually, ESQPTs are linked to QPTs; below the critical parameter $\lambda_{c}$ no transition appears, while above $\lambda_{c}$ the nonanalytic behavior characteristic of the QPT propagates to a certain critical excitation energy $E_{c}$. However, in some cases an ESQPT occurs without the corresponding QPT. As a paradigmatic example, we mention the realization of the Lipkin-Meshkov-Glick model studied in [6].

ESQPTs entail dramatic dynamic consequences, like the enhancement of the decoherence if the ESQPT takes place in the environment with which a quantum system interacts [9], the onset of chaos [10], or the emergence of symmetry-breaking equilibrium states after a quench [11]. They appear in models pertaining to different branches of physics, like the interacting boson model (IBM) [12,13], Lipkin-Meshkov-Glick model [14], vibron model [15], Dicke and Jaynes-Cummings models $[10,16,17]$, kicked top [18], and microwave Dirac billiards [19]. It is worth mentioning that, despite the fact that the excitation energy is linked to the temperature of an isolated system, ESQPTs are qualitatively different from thermal phase transitions. The existence of a critical energy does not necessarily entail a corresponding critical temperature. For example, the Dicke model restricted to the maximum angular momentum sector displays an ESQPT, but has no thermal phase transition [20].

In order to investigate these issues, we treat two related systems. The first consists of an atomic Bose gas trapped in a two-well potential, and the second incorporates the interaction of the atoms with a diatomic molecule. Mixed condensates of atoms and molecules have been experimentally realized by means of the manipulation of a Feshbach resonance [21-23]. Mixtures of atoms and molecules in double-well potentials have been studied previously in [24]. In [25] a similar system was used to model the atom-atom interaction by means of a Feshbach resonance.

The organization of the paper is as follows. We introduce the two models in Sec. II. In Secs. III and IV the ground state quantum phase transitions are investigated numerically as well as analytically for both models, first, within a mean-field 
approach, for which we later incorporate fluctuations. Section $\mathrm{V}$ is devoted to analyze ESQPTs in both models. Finally, in Sec. VI the main conclusions of this work are summarized.

\section{MODELS}

We shall study a bosonic atom gas confined in a double-well potential as a model of a bosonic Josephson junction [26]. It has been recently shown that a restriction to the lowest two modes is a valid approximation for a wide range of the bosonic interaction [4]. In this limit, the model reduces to the two-site Bose-Hubbard Hamiltonian. Each site represents the lowest mode of the right and left wells, respectively,

$$
H_{1}=-J\left(a_{L}^{\dagger} a_{R}+a_{R}^{\dagger} a_{L}\right)+\frac{U}{N}\left(a_{L}^{\dagger} a_{L}^{\dagger} a_{L} a_{L}+a_{R}^{\dagger} a_{R}^{\dagger} a_{R} a_{R}\right)
$$

where the $a_{k}^{\dagger}$ and $a_{k}$ ( $k=L, R$ for left and right wells) operators are the atom creation and annihilation operators in each well and obey the usual boson commutation rules, $N$ is the total number of atoms, $J$ is the hopping strength, and $U$ is atomic interaction. Note that the scaling factor $1 / N$ in front of the atomic interaction is needed to ensure that the energy per particle $E / N$ is well defined in the thermodynamic limit. The static and dynamic properties of the Hamiltonian (1) have been extensively studied in recent years [27-30]. Moreover, it has been shown that (1) is a quantum integrable model and a particular limit of Richardson-Gaudin bosonic families [31-33].

In addition to (1), we will consider here an extension of the two-site Hubbard model by the explicit inclusion of an interacting diatomic molecule. The resulting two-channel Hamiltonian is

$$
\begin{aligned}
H_{2}= & -J\left(a_{L}^{\dagger} a_{R}+a_{R}^{\dagger} a_{L}\right)+\frac{U}{N}\left(a_{L}^{\dagger} a_{L}^{\dagger} a_{L} a_{L}+a_{R}^{\dagger} a_{R}^{\dagger} a_{R} a_{R}\right) \\
& +\omega b^{\dagger} b-\frac{g}{\sqrt{2 N}}\left[b^{\dagger}\left(a_{L} a_{L}+a_{R} a_{R}\right)+\left(a_{L}^{\dagger} a_{L}^{\dagger}+a_{R}^{\dagger} a_{R}^{\dagger}\right) b\right]
\end{aligned}
$$

where $N$ is the total number of atoms plus twice the number of molecules, $N=2 b^{\dagger} b+\left(a_{L}^{\dagger} a_{L}+a_{R}^{\dagger} a_{R}\right)$, and $b^{\dagger}(b)$ creates (annihilates) a molecule. The operators $b$ and $b^{\dagger}$ follow the usual bosonic commutation rules. Because of the shape of the atom-molecule interaction (two atoms are destroyed to create a molecule, and vice versa) they represent a dimeric entity, giving rise to the previously written conservation of the total number of particles $N$. The frequency $\omega$ represents the selfenergy of the molecule. When it goes to zero, the Hamiltonian (2) describes a Feshbach resonance interacting with the atomic cloud. In what follows we will use the hopping term $J$ as the energy unit. This Hamiltonian is in general nonintegrable. However, as a function of the interaction parameters $\omega, U$, and $g$ it has two integrable limits which will allow us to explore the transition from regularity to chaos. As in the previous case, the scaling factors $1 / N$ and $1 / \sqrt{N}$ in front of the atom-atom and the atom-molecule interaction ensure that the energy per particle is well defined in the thermodynamic limit.
For both Hamiltonians, (1) and (2), $N$ is a conserved quantity. They also conserve a kind of parity, related to the interchange between atoms in left and right wells.

In connection with this symmetry, it is worth mentioning that the population imbalance operator that counts the difference between the number of atoms in the left and right wells,

$$
\hat{I}=a_{R}^{\dagger} a_{R}-a_{L}^{\dagger} a_{L}
$$

does not commute with the parity operator. Therefore, its expectation value is zero in any common eigenstate of the Hamiltonian and the parity operator.

\section{PHASE DIAGRAM}

In this section we analyze and compare the ground state phase diagram of both models, the double well with and without the interaction with the diatomic molecule. In order to carry out this comparison, we select the atomic interaction $U$ that is common to both models as the control parameter, and keep fixed $\omega$ and $g$. As it will be seen below, the ground state of one of the phases is doubly degenerate due to spontaneous symmetry breaking. Thus, the population imbalance is a good candidate of order parameter for this transition. In fact, it has been used in [4] to characterize the macroscopic self-trapping in the two-site Bose-Hubbard model. However, it is not possible to rely directly on the population imbalance to study numerically this transition, because both Hamiltonians (1) and (2) have a well defined parity and, therefore, the imbalance expectation value is zero in any of their eigenstates. One possible solution is to introduce a small symmetry-breaking term to break the degeneracies, as has been done in [4]. In this work we do not follow this way; we will use, instead, a new order parameter derived from a quantum version of the Fisher information, defined as [34]

$$
F_{Q F I}=\left\langle\left(\widehat{n}_{R}-\widehat{n}_{L}\right)^{2}\right\rangle-\left(\left\langle\widehat{n}_{R}-\widehat{n}_{L}\right\rangle\right)^{2} .
$$

For eigenstates with well-defined parities, this expression reduces to

$$
F_{Q F I}=\left\langle\left(\widehat{n}_{R}-\widehat{n}_{L}\right)^{2}\right\rangle .
$$

Therefore, to obtain a quantity proportional to the number of left- or right-well atoms, we will use $\sqrt{F_{Q F I}}$ as the order parameter of the transition. As will be seen below, this magnitude behaves in the same way as the population imbalance in the exact solution of both Hamiltonians.

\section{A. Mean-field approximation}

The mean-field or semiclassical approximation is expected to provide accurate results for this kind of two-level systems in the large $N$ limit. In fact, it has been shown that the ground state energy in this approximation is exact to leading order in $N$ [35]. The great advantage of this approach is that it can be solved analytically. We use boson coherent states as variational wave functions to model the ground state of both Hamiltonians. The corresponding wave functions are

$$
\left|\Psi_{1}\right\rangle=e^{\sqrt{N}\left(\gamma_{R} a_{R}^{\dagger}+\gamma_{L} a_{L}^{\dagger}\right)}|0\rangle
$$


for the case without molecule, and

$$
\left|\Psi_{2}\right\rangle=e^{\sqrt{N}\left[(\beta / 2) b^{\dagger}+\gamma_{R} a_{R}^{\dagger}+\gamma_{L} a_{L}^{\dagger}\right]}|0\rangle,
$$

for the case with molecule. They are eigenstates of the annihilation operators $a_{L}, a_{R}$ (both) and $b$ (only the last), with eigenvalues $\sqrt{N} \gamma_{R}, \sqrt{N} \gamma_{L}$, and $\sqrt{N} \beta / 2$, respectively; hence, they fulfill the mathematical requirement for coherent states. The former could be obtained from the second one as the limit in which the atom-molecule coupling parameters $g$ and the corresponding amplitude in the coherent state $(\beta)$ go to zero, and the self-energy of the molecule $\omega$ goes to infinity: $g \rightarrow 0, \omega \rightarrow \infty$ and $\beta \rightarrow 0$. Therefore we will mostly concentrate on the second model, and treat the other one as a particular case in the appropriate limit. It is important to note that both coherent states break the parity symmetry and will give a finite population imbalance different from zero in the symmetry-broken phase.

The energy per particle using the coherent state $\left|\Psi_{2}\right\rangle$ is

$$
\begin{aligned}
\frac{E}{N}= & \frac{\left\langle\Psi_{2}\left|H_{2}\right| \Psi_{2}\right\rangle}{N\left\langle\Psi_{2} \mid \Psi_{2}\right\rangle}=-2 J \gamma_{R} \gamma_{L}+\frac{\omega}{2} \beta^{2}-g \beta\left(\gamma_{R}^{2}+\gamma_{L}^{2}\right) \\
& +U\left(\gamma_{R}^{4}+\gamma_{L}^{4}\right)
\end{aligned}
$$

where the variational parameters are assumed to be real, provided $g>0$. By minimizing this energy surface for the corresponding set of parameters $J, \omega, g$, and $U$, optimal variational parameters $\beta, \gamma_{R}$, and $\gamma_{L}$ are obtained, providing an approximation to the exact ground state wave function and energy. This method is only exact, as mentioned above, for the leading order in $N$ [35]. Because of the conservation of the total number of particles in the system, there is a constraint for the variational parameters: $\beta^{2}+\gamma_{R}^{2}+\gamma_{L}^{2}=1$. This condition is included in the minimization by using a Lagrange multiplier which introduces a new parameter, $\lambda$.

When the energy surface, with the constraint of the particle number conservation, is minimized, the optimal values of the variational parameters as a function of the control parameters are determined from the set of equations,

$$
\begin{aligned}
\beta^{2}+\gamma_{R}^{2}+\gamma_{L}^{2} & =1, \\
-g\left(\gamma_{R}^{2}+\gamma_{L}^{2}\right)+\omega \beta-2 \lambda \beta & =0, \\
g \beta \gamma_{R}-2 U \gamma_{R}^{3}+J \gamma_{L}+\lambda \gamma_{R} & =0, \\
J \gamma_{R}+g \beta \gamma_{L}-2 U \gamma_{L}^{3}+\lambda \gamma_{L} & =0 .
\end{aligned}
$$

This set of four nonlinear equations has two classes of solutions: (a) the symmetric, with $\gamma_{R}=\gamma_{L}=\gamma$, and the nonsymmetric, (b) $\gamma_{R} \neq \gamma_{L}$. The former corresponds to the symmetric phase; the latter, to the nonsymmetric phase.

The solutions in the symmetric phase are

$$
\beta= \begin{cases}-\frac{g}{2 U}+\frac{(1-i \sqrt{3}) A}{6 \sqrt[3]{4} U\left(B+\sqrt{B^{2}+4 A^{3}}\right)^{1 / 3}}-\frac{(1+i \sqrt{3})\left(B+\sqrt{\left.B^{2}+4 A^{3}\right)^{1 / 3}}\right.}{12 \sqrt[3]{2} U}, & \text { if } \quad U \in\left(U_{c}, 0\right), \\ \frac{-2 J-\omega+\sqrt{12 g^{2}+(2 J+\omega)^{2}}}{6 g}, & \text { if } \quad U=0, \\ -\frac{g}{2 U}-\frac{A}{3 \sqrt[3]{4} U\left(B+\sqrt{B^{2}+4 A^{3}}\right)^{1 / 3}}+\frac{\left(B+\sqrt{B^{2}+4 A^{3}}\right)^{1 / 3}}{6 \sqrt[3]{2} U}, & \text { if } U \in(0, \infty), \\ \gamma=\sqrt{\frac{1-\beta^{2}}{2},} & \end{cases}
$$

with

$$
\begin{aligned}
& A=-9 g^{2}+6 U(2 J-2 U+\omega), \\
& B=54 g\left(2 J U-g^{2}+U \omega\right) .
\end{aligned}
$$

The range of variation of the Hamiltonian parameters for this solution is $g \in \mathbb{R}-\{0\}, \omega$ and $J \in \mathbb{R}$. $U_{c}$ is the critical value for which the system undergoes the QPT.

For the nonsymmetric phase, $U \in\left(-\infty, U_{c}\right)$, the solutions are

$$
\begin{gathered}
\beta=-\frac{g}{4 U}+\frac{(1-i \sqrt{3}) A^{\prime}}{12 \sqrt[3]{2} U\left(B^{\prime}+\sqrt{\left(B^{\prime}\right)^{2}+4\left(A^{\prime}\right)^{3}}\right)^{1 / 3}} \\
-\frac{(1+i \sqrt{3})\left(B^{\prime}+\sqrt{\left(B^{\prime}\right)^{2}+4\left(A^{\prime}\right)^{3}}\right)^{1 / 3}}{24 \sqrt[3]{2} U} \\
\gamma_{R}=\sqrt{\frac{1-\beta^{2}}{2}+\frac{\sqrt{-J^{2}+U^{2}\left(\beta^{2}-1\right)^{2}}}{2 U}}
\end{gathered}
$$

$$
\gamma_{L}=-\frac{J}{2 U \gamma_{R}}
$$

with

$$
\begin{aligned}
& A^{\prime}=12 U(\omega-4 U)-9 g^{2}, \\
& B^{\prime}=54 g\left(2 U \omega-g^{2}\right) .
\end{aligned}
$$

For this solution the Hamiltonian parameters are restricted to $g \in \mathbb{R}-\{0\}, \omega \in \mathbb{R}$ and $J \in \mathbb{R}-\{0\}$.

The critical value of $U\left(U_{c}\right)$ can be computed using Eqs. (13) and (17). The variational parameter $\beta$ has to be a continuous function of $U$ to ensure the continuity of the ground state energy. By equating both expressions of $\beta$ in the symmetric, (13), and nonsymmetric, (17), phases we can determine numerically the critical value $U_{c}$. For instance, for a system defined by $J=1, \omega=5$, and $g=5$, the value obtained for $U_{c}$ is $U_{c}=-1.14018$.

A similar analysis can be performed for the limit without coupling to the molecule obtaining the following expressions 
for the variational parameters:

$$
\begin{aligned}
& \gamma_{R}= \begin{cases}\sqrt{\frac{1}{2}+\operatorname{sgn}(U) \frac{\sqrt{U^{2}-1}}{2 U}}, & \text { if } U<U_{c}, \\
\frac{1}{\sqrt{2}}, & \text { if } U \geqslant U_{c},\end{cases} \\
& \gamma_{L}= \begin{cases}\frac{-J}{2 U} \frac{1}{\sqrt{\frac{1}{2}+\operatorname{sgn}(U) \frac{\sqrt{U^{2}-1}}{2 U}},} & \text { if } U<U_{c}, \\
\frac{1}{\sqrt{2}}, & \text { if } U \geqslant U_{c} .\end{cases}
\end{aligned}
$$

In this case, the critical value for the coupling constant is $U_{c}=-1$.

Note that Eqs. (9)-(12) are invariant under the transformation $\gamma_{L} \rightarrow-\gamma_{L}, \gamma_{R} \rightarrow-\gamma_{R}$, which implies that there always exist two variational solutions. In the symmetric phase, both of them give rise to $\widehat{I}=0$, corresponding to the same physical state. On the contrary, in the nonsymmetric phase both have nonzero imbalance, $\widehat{I_{1}}=-\widehat{I_{2}}$, implying that the ground state is degenerate. So, in this case any linear combination of these two solutions is also a ground state of the system, and thus it is possible to build a parity-projected solution, with $\widehat{I}=0$. As a consequence, the critical coupling $U_{c}$ separates two different regions: the symmetric one, in which the populations of the two wells are always equal, and the nonsymmetric one, in which one can find symmetry-breaking ground states. As the coherent states used in this section break the symmetry for $\gamma_{L} \neq \gamma_{R}$, they provide an accurate description of the transition in terms of the imbalance.

\section{B. Numerical results}

In this subsection, we compare the mean-field results with exact diagonalizations for finite systems. In Figs. 1(a) and 1(c) we depict the behavior of the variational parameters $\gamma_{R}$ and $\gamma_{L}$ for both cases, without molecule (right panels) and with molecule (left panels). It is clearly seen that $\gamma_{L}$ and $\gamma_{R}$ are equal to each other in one of the phases, and different in the other, for both models. In panels (b) and (d) of the figure we plot the mean-field result for the imbalance, together with the numerical values for $\sqrt{F_{Q F I}}$, obtained for $N=3200$ particles in the case without molecules, and with $N=100$ and $N=200$ particles in the case with molecule. The imbalance behaves as a typical order parameter: it is zero in one of the phases, and becomes nonzero after crossing the critical point $U_{c}$, showing a nonanalytic behavior at $U_{c}$. Therefore, the symmetric phase is characterized by the same population of atoms in the two wells, whereas in the nonsymmetric phase there is a finite imbalance. Numerical results show that $\sqrt{F_{Q F I}}$ provides also a correct description of the QPT: it is nonzero in the nonsymmetric phase, and tends to zero in the symmetric one. Note that the Fisher information measures the fluctuations around the equilibrium state, and therefore it cannot be strictly zero in finite systems. However, we can see from the figure how this signature tends to zero as the size of the system increases.

In Fig. 2 we depict the energy (per particle) difference between the ground state and the first-excited state for both the case with (a) and without (b) molecule, for different system sizes. For the former, we have used $N=20,40,80,160$, and 320 particles; for the second one $N=250,1000,4000$, and 16000. Dotted vertical lines show the mean-field values for the critical coupling $U_{c}$. Solid horizontal lines show a numerical bound to distinguish the degenerate from the nondegenerate phase. The value of the coupling for which the gap becomes less than this bound is the finite-size precursor of the transition $U_{c}^{(N)}$. Obviously, the precise value of this precursor depends on the chosen value for the bound, but the important result is the finite-size scaling and the fact that $U_{c}^{(N)} \rightarrow U_{c}$ in the thermodynamic limit. From our numerical results, it seems clear that the finite-size precursor of the transition tends to the mean-field critical behavior in the thermodynamic limit.

In Fig. 3 we show how the finite-size precursor $U_{c}^{(N)}$ scales with the system size, in a double-logarithmic scale, both for the case with (a) and without (b) molecule. It is clearly seen that $U_{c}^{(N)}-U_{c} \rightarrow 0$, when $N \rightarrow \infty$. In both cases a scaling
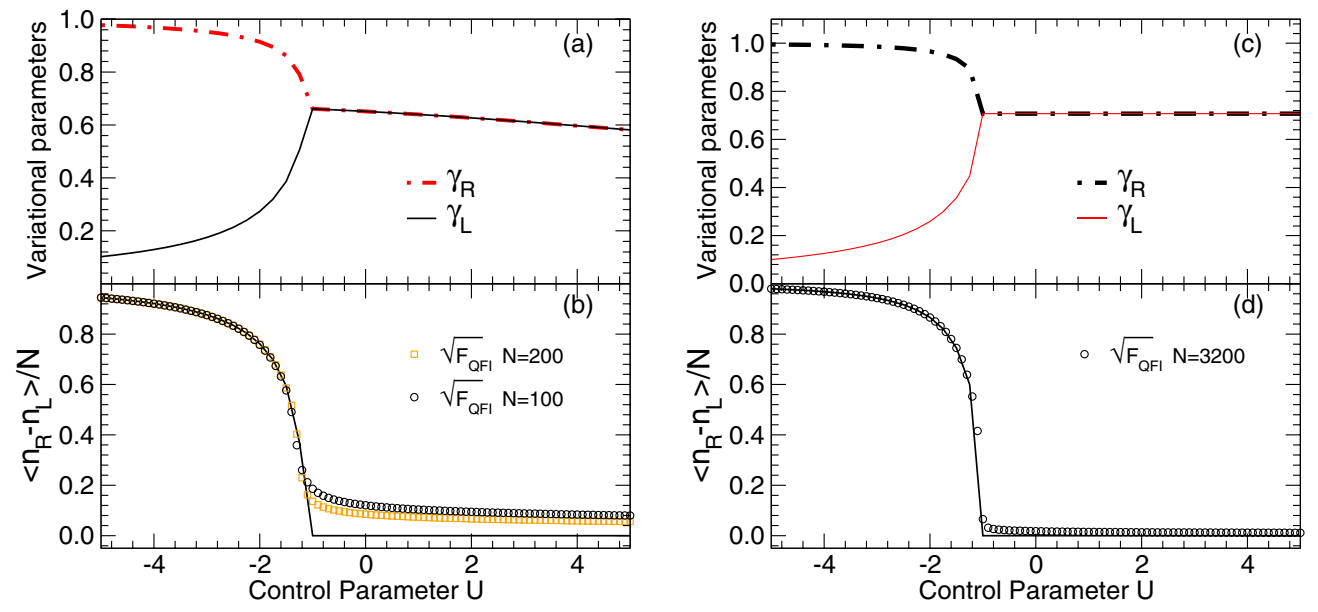

FIG. 1. (Color online) Panels (a) and (c) show the variational parameters $\gamma_{R}$ and $\gamma_{L}$, as a function of the control parameter $U$. Panels (b) and (d) show the normalized expectation value of the invalance operator $\widehat{I}$ in solid line, and the square root of the Fisher information, obtained by numerical diagonalization, in open symbols. Panels (a) and (b) are for the mixed atom-molecule system [two numerical calculations for different system sizes are shown in (b), (black) circles correspond to $N=100$, and (orange) squares, to $N=200]$ whereas panels (c) and (d) present the system without molecule. 

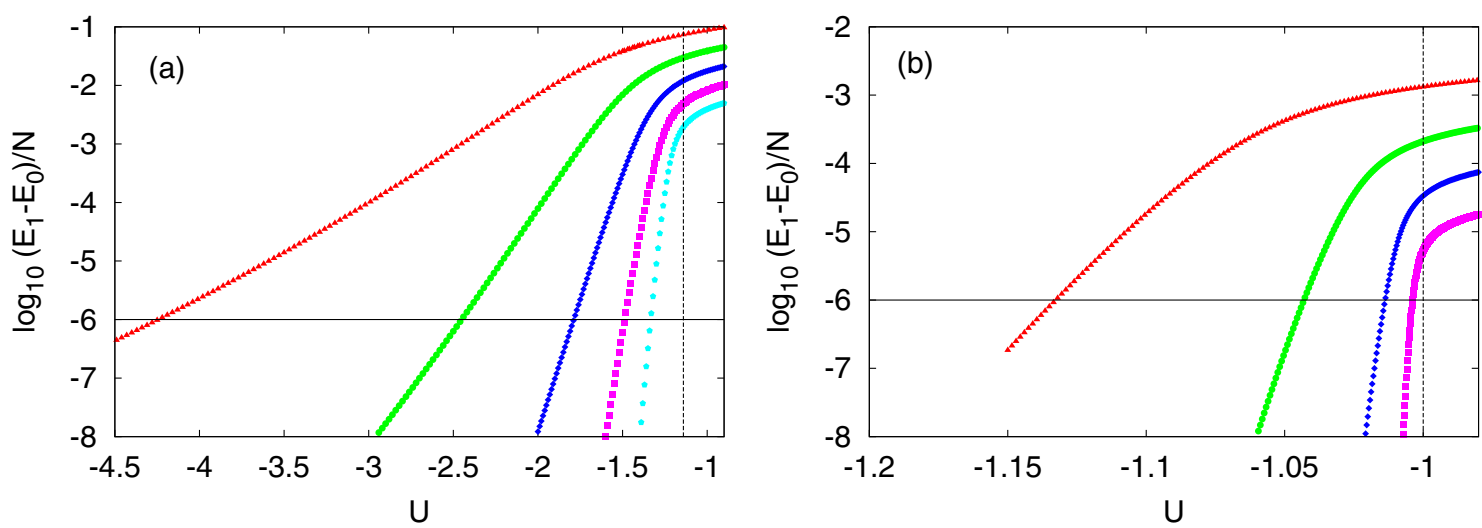

FIG. 2. (Color online) Gap between ground state and first-excited state, for different system sizes: system with molecule, $N=20$ (triangles, red online), 40 (circles, green online), 80 (diamonds, blue online), 160 (squares, magenta online) and 320 (hexagons, cyan online), in panel (a); and system without molecule, $N=250$ (triangles, red online), 1000 (circles, green online), 4000 (diamonds, blue online) and 16000 (squares, magenta online), panel (b). In all the cases, system sizes increase from left to right.

power law $U_{c}^{(N)}-U_{c} \propto N^{-\alpha}$ is found, with $\alpha=0.84 \pm 0.03$ for the case with molecule, and $\alpha=0.99 \pm 0.02$ for the case without molecule.

The results presented in the subsection show that a secondorder quantum phase transition takes place at a critical coupling $U_{c}$, for both models. The main difference between them resides in the finite-size scaling exponent $\alpha$.

\section{LOW ENERGY SPECTRUM}

\section{A. Beyond mean-field approximation}

The mean-field results obtained in the preceding section provide the leading order $N$ description of the models. In order to improve the mean-field description, we first perform a shift transformation to the $a_{L}, a_{R}$, and $b$ bosons, defining the shifted bosons $d_{0}, d_{1}$, and $f$

$$
\begin{aligned}
& a_{R}^{\dagger}=\sqrt{N} \gamma_{R}+d_{0}^{\dagger}, \\
& a_{L}^{\dagger}=\sqrt{N} \gamma_{L}+d_{1}^{\dagger}, \\
& b_{L}^{\dagger}=\sqrt{N / 2} \beta+f^{\dagger},
\end{aligned}
$$

where $\gamma_{R}, \gamma_{L}$, and $\beta$ are taken as real variational parameters, and $N$ is the total number of particles in the system. These new bosons, $d_{i}$ and $f$, satisfy the usual bosonic commutation relations.

When this shift transformation is introduced into the Hamiltonian, an expansion of $\widehat{\mathcal{H}}$ in powers of $N$ is obtained,

$$
\widehat{\mathcal{H}}=\widehat{H}_{1}+\widehat{H}_{1 / 2}+\widehat{H}_{0}+\mathcal{O}(1 / \sqrt{N}),
$$

where $\widehat{H}_{i}$ represents the i-esim term of the expansion, which is proportional to the power $N^{i}$.

The first term, $\widehat{H}_{1}$, is the mean-field energy already computed in the previous section, with $\gamma_{R}, \gamma_{L}$, and $\beta$ evaluated at the energy minimum. The next order in the Hamiltonian expansion, $\widehat{H}_{1 / 2}$, is

$$
\begin{aligned}
\widehat{H}_{1 / 2}= & \sqrt{N}\left[\frac{-1}{\sqrt{2}}\left(\gamma_{R}^{2}+\gamma_{L}^{2}\right) g+\beta(2 \lambda-\omega) f\right. \\
& \left.-\left[J \gamma_{L}+(\lambda+\beta g) \gamma_{R}-2 U \gamma_{R}^{3}\right]\left(d_{0}+d_{1}\right)+\text { H.c. }\right] .
\end{aligned}
$$

This term cancels at the equilibrium values of the variational parameters. This stems from the relation $\widehat{H}_{1 / 2}=$ $\sum_{i} \frac{1}{2 \sqrt{N}} \frac{d \widehat{H}_{1}}{d \gamma_{i}}\left(d_{i}+d_{i}^{\dagger}\right)+\frac{1}{\sqrt{2 N}} \frac{d \widehat{H}_{1}}{d \beta}\left(f+f^{\dagger}\right), i=0,1$. Thus, the first nonzero correction to the mean-field energy comes from
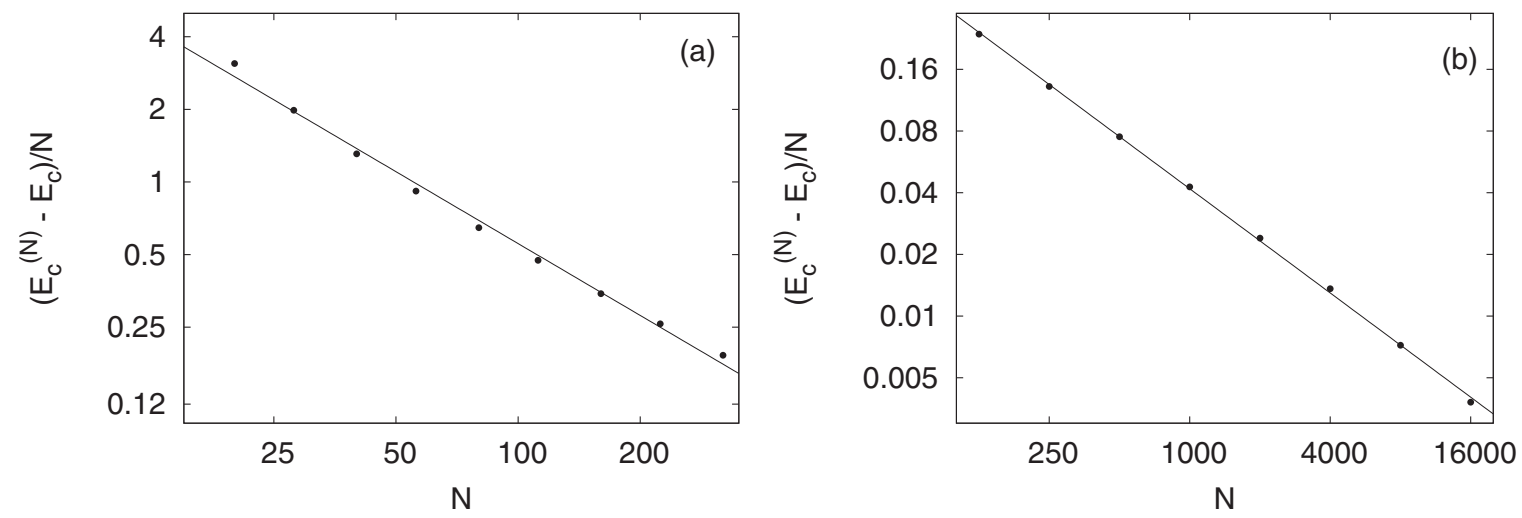

FIG. 3. Scaling of the finite-size precursor of the critical point. 
the $\widehat{H}_{0}$ term. This term can be expressed in compact form as

$$
\widehat{H}_{0}=\frac{1}{2} \delta^{\dagger} M \delta-\frac{1}{2} \operatorname{Tr} Y,
$$

where $\delta$ and $\delta^{\dagger}$ are column and row vectors of dimension 6 respectively, $M$ is a $6 \times 6$ matrix, and $Y$ and $Z$ are $3 \times 3$ matrices. $M$ has the form

$$
M=\left(\begin{array}{ll}
Y & Z \\
Z & Y
\end{array}\right)
$$

with the $3 \times 3 Y$ and $Z$ matrices given by

$$
\begin{aligned}
& Y=\left(\begin{array}{ccc}
4 U \gamma_{R}^{2}-\lambda & -J & -\sqrt{2} \gamma_{R} g \\
-J & 4 U \gamma_{L}^{2}-\lambda & -\sqrt{2} \gamma_{L} g \\
-\sqrt{2} \gamma_{R} g & -\sqrt{2} \gamma_{L} g & \omega-2 \lambda
\end{array}\right), \\
& Z=\left(\begin{array}{ccc}
2 U \gamma_{R}^{2}-\beta g & 0 & 0 \\
0 & 2 U \gamma_{L}^{2}-\beta g & 0 \\
0 & 0 & 0
\end{array}\right), \\
& \delta^{\dagger}=\left(\begin{array}{llllll}
d_{0}^{\dagger} & d_{1}^{\dagger} & f^{\dagger} & d_{0} & d_{1} & f
\end{array}\right) .
\end{aligned}
$$

The quadratic Hamiltonian (29) in the shifted bosons $d$ and $f$ can be diagonalized by means of a canonical transformation [36], which is equivalent to diagonalize the matrix

$$
\tilde{M}=\left(\begin{array}{rr}
Y & Z \\
-Z & -Y
\end{array}\right)
$$

The matrix $\tilde{M}$ has a Goldstone mode at zero energy due to the breaking of the U(1) symmetry associated with the condensation phenomenon. It also has two real and positive eigenvalues describing the excited states of the system. Once the canonical transformation is defined through the diagonalization of $\tilde{M}, \widehat{H}_{0}$ can be expressed as

$$
\widehat{H}_{0}=\sum_{i=1}^{3} g_{i}^{\dagger} g_{i} \Delta_{i}+\frac{1}{2} \sum_{i=1}^{3} \Delta_{i}-\frac{1}{2} \operatorname{Tr} Y,
$$

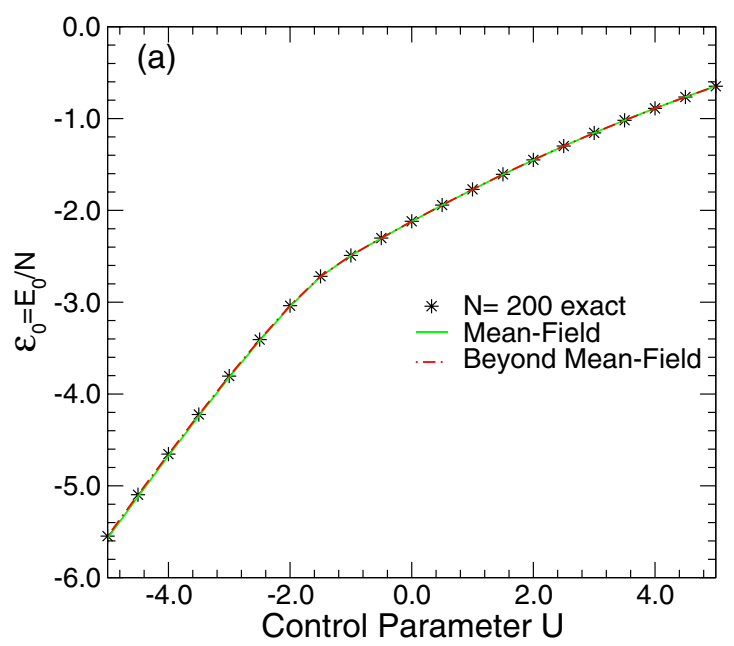

where $g_{i}\left(g_{i}^{\dagger}\right)$ are the new quasiparticle operators, and $\Delta_{i}$ $(i=1,2,3)$ are the corresponding eigenvalues of $\tilde{M}$. This problem has no analytic expression for $\Delta_{i}$ in general. For a given set of Hamiltonian parameters $g, \omega$, and $J$, we diagonalize numerically $\tilde{M}$ to compute the eigenvalues $\Delta_{i}$. Notice that the term $\frac{1}{2} \sum_{i=1}^{3} \Delta_{i}-\frac{1}{2} \operatorname{Tr} Y$ gives a correction for the ground state mean-field energy.

The limit with no molecule is obtained from the analytic expressions for the mean-field corrections when $g \rightarrow 0$ and $\omega \rightarrow \infty$. Notice that the $f\left(f^{\dagger}\right)$ modes decouple from the $g_{i}$ $\left(g_{i}^{\dagger}\right)$ modes and its energy goes to infinity. In this limit $\tilde{M}$ becomes a $4 \times 4$ matrix and its eigenvalues provide the two different excited modes of the model. One of these modes is always zero, corresponding to the Goldstone mode associated with the breaking of $\mathrm{U}(1)$ symmetry. The reduction of the $\tilde{M}$ matrix size allows for the derivation of analytic expressions for these modes since its characteristic polynomial has degree 4 and can be solved by radicals.

\section{B. Comparison with numerical calculations}

In order to check the quality of the mean-field approach and the improvement provided by the corrections to the mean-field approximation discussed in the preceding subsection, we present here a comparison with the exact diagonalization of (2) for finite systems. The ground state energy per particle versus the control parameter $U$, for fixed values of the other parameters $J=1, \omega=5$, and $g=5$, is shown in Fig. 4(a). The stars represent the exact numerical calculation for a system with $N=200$ bosons. The thin full line is the mean-field result, while the dot-dashed line corresponds to the beyond mean-field approximation. For this system size the differences between the exact results and the two approximations are imperceptible at the panel (a) scale. In order to see the numerical differences, we present in panel (b) the differences between the mean-field and beyond mean-field calculations with the exact numerical results in a larger scale. The $U$ values

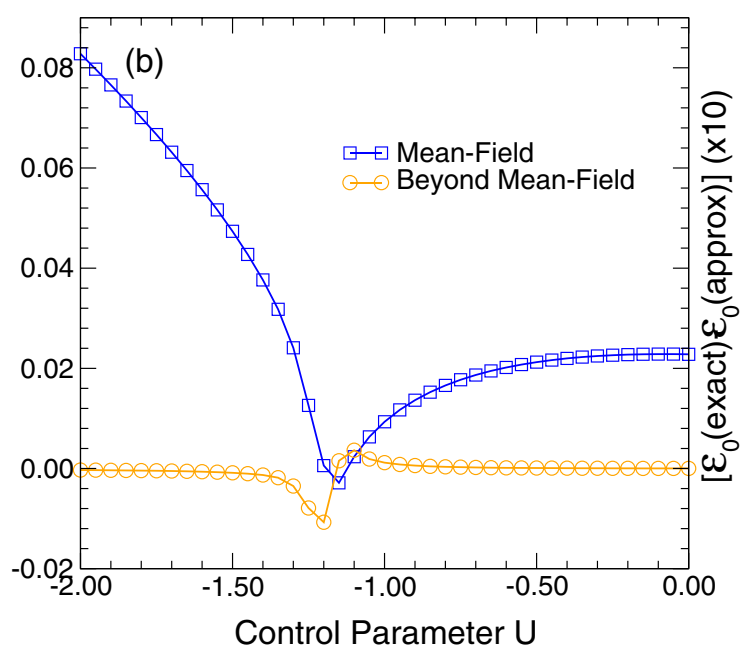

FIG. 4. (Color online) In panel (a) the ground state energy per particle of the model with the diatomic is plotted as a function of the control parameter $U$ for $N=200$. Panel (b) shows the difference between the exact ground state energy per particle obtained numerically and the values obtained by using the two analytic approximations considered in this work in a small region around the critical point for a system with $N=200$. The rest of the control parameters in the Bose-Hubbard Hamiltonian are $J=1, \omega=5$, and $g=5$. 

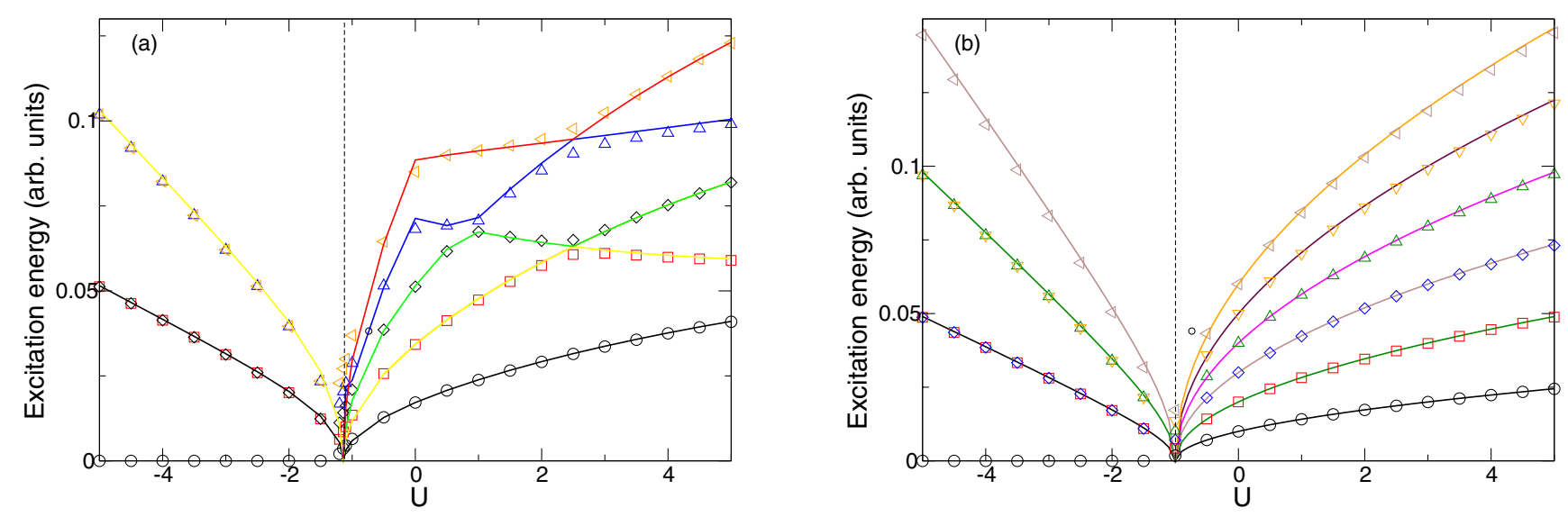

FIG. 5. (Color online) First few excited-state energy gaps as a function of the control parameter $U$. Symbols are the exact results for $N=200$, while lines provide the beyond mean-field approximation. The rest of the parameters are fixed to $J=1, \omega=5$, and $g=5$ in panel (a), and $J=1$ in panel (b). The vertical dotted line marks the separation between the two phases.

in (b) are restricted to the region around the critical point at $U_{c} \sim-1.14$. As apparent in Fig. 4 (b), the computed ground state energies from beyond mean field clearly improve the mean-field results. The beyond mean-field calculations match the exact results in most of the range of variation of the control parameter; the differences with the exact numerical results are concentrated in a small region close to the critical point. This validates the beyond mean-field formalism as a valuable tool for taking into account the finite-size effects in these models.

Figure 5 shows the lowest excited energies obtained by means of the beyond mean-field treatment (full lines), compared with the exact numerical results (symbols), for both the case with (a) and without (b) the diatomic molecule. In both cases, the beyond mean-field approach gives a very accurate description of the low-energy spectra, including the important fact that the energy levels are degenerate in pairs in one of the phases, while this degeneracy is broken in the other. The main difference between the two systems lay in the qualitative behavior of the levels. In the case without the molecule, the spectrum is similar in both phases, though it seems more compressed in the symmetric one. On the contrary, both phases show clear qualitative differences in the case with the molecule. The spectrum of the symmetry-broken phase is very smooth, all the levels changing mildly with energy. The symmetric phase is characterized by an erratic behavior of the levels, with crossings between levels with different parities, and multiple avoided crossings. This constitutes a signature of a highly complex behavior related to the onset of chaos (see Sec. V for more details).

\section{EXCITED-STATE QUANTUM PHASE TRANSITIONS}

In some collective many-body quantum systems, QPTs are accompanied by ESQPTs, giving rise to a critical energy $E_{c}$ for certain values of the system control parameter [9-17]. Traditionally, ESQPTs have been linked to singularities in the density of states or in one of its derivatives, depending on the number of system's degrees of freedom in the semiclassical limit [37]. It has been shown that for a system with just one effective degree of freedom, as the Tavis-Cummings model, a $\lambda$ singularity in the density of states characterizes the ESQPT.
For the Dicke model, which has two effective degrees of freedom, the singularity making the ESQPT appears in the first derivative of the density of states [17]. This fact entails an increasing difficulty for detecting such a critical behavior in complex systems, with many effective degrees of freedom.

In this work, we rely on a different way to identify ESQPTs. In general, the critical energy separates two different regions of the spectrum which share some of the properties of the ground state at one and at either side of the QPT. For instance, in Dicke and Lipkin models the critical energy separates one region in which all eigenstates are doubly degenerate from another in which there are no degeneracies and all the levels have a well defined parity [11]. So, in order to characterize the ESQPTs in our system, we study the distance between consecutive energy levels with opposite parity. We follow the same criterion as in Fig. 2. Figure 6 shows the energy per particle versus the strength of the $U$ parameter for the two systems. Panel (a) corresponds to the case with a diatomic molecule and a total of $N=320$ particles, with $J=1, \omega=5$, and $g=5$; while panel (b) corresponds to the case without molecule, with $N=1000$ particles and $J=1$. The lowest line is the ground state energy, while the uppermost line gives the limit of the calculated spectra for the system size used (highest calculated excited state). There are many excited states in between both lines. In both panels two vertical dotted lines are plotted: the left line gives the ground state QPT, $U_{c}$, while the right one marks an equivalent singularity for the highest excited state, $\widetilde{U}_{c}$. The energy region with no degeneracies is called normal (light filled region, yellow online), while the region with double degeneracies is a reflection of the appearance of a broken-symmetry (dark filled region, blue online). Our calculations clearly identify an ESQPT, that is marked by the line in between light and dark regions. It separates normal (nondegenerate) from broken-symmetry (degenerate) phases. Figure 6 shows that both systems behave in the same qualitative way. For $U<U_{c}$, the lower part of the spectrum is degenerate, whereas the upper part is not degenerate. For large values of the control parameter, $U>\widetilde{U}_{c}$, the spectrum behaves in the opposite way: the upper part is degenerate, whereas the lower part is not. In between, $U_{c} \leqslant U \leqslant \widetilde{U}_{c}$, all the energy levels are not degenerate. This entails that, for attractive interactions 

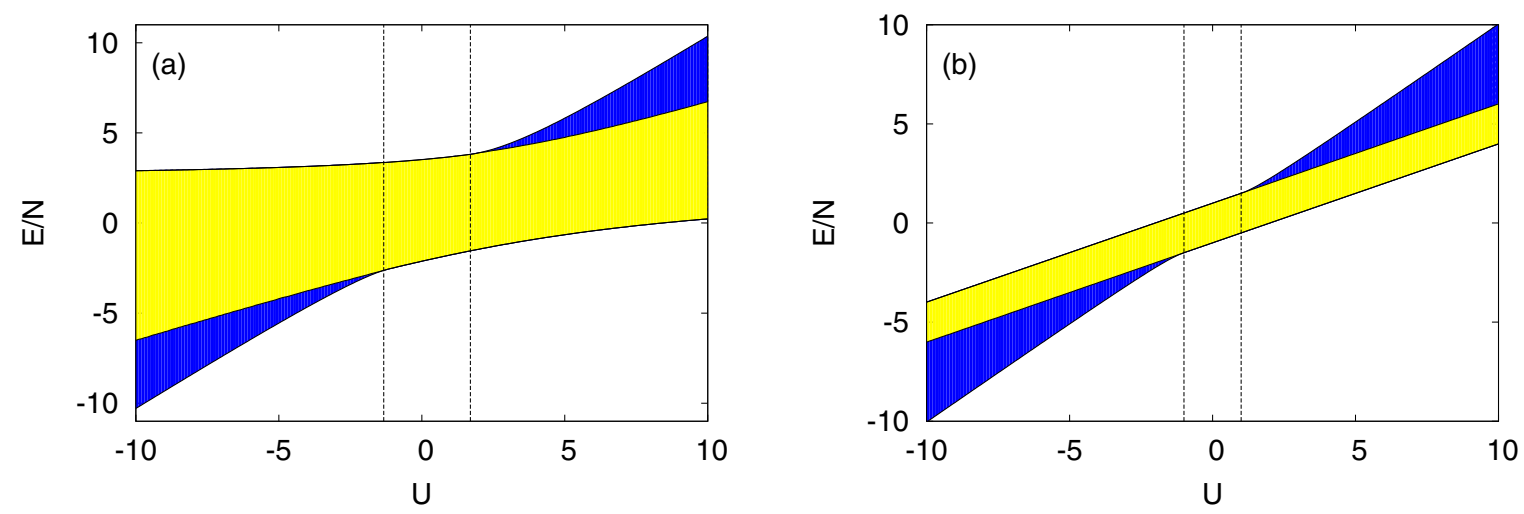

FIG. 6. (Color online) Complete phase diagram. Panel (a) depicts the case with molecule, obtained with $N=320$ particles, and $J=1$, $\omega=5$, and $g=5$. Panel (b) depicts the case without molecule, with $N=1000$ particles and $J=1$. In both cases, the dark filled region (blue online) indicates the symmetry-breaking phase, in which the eigenstates are doubly degenerate, and the light filled region (yellow online) is the normal phase, in which there are no degeneracies and the parity is a good quantum number for all the eigenstates. Vertical dotted lines enclose the region in which there is no ESQPT.

$U<0$ there is a QPT at a critical coupling $U_{c}$, and a critical energy $E_{c}$ for $U<U_{c}$. On the contrary, if the interaction is repulsive $U>0$, an analog of a QPT takes place on the highest-excited level at a critical coupling $\widetilde{U}_{c}$, and also a critical energy appears for $U>\widetilde{U}_{c}$. No ESQPT is observed in the region $U_{c} \leqslant U \leqslant \widetilde{U}_{c}$.

The critical line can be easily estimated from the numerics in the case without the molecule and gives

$$
E_{c}= \begin{cases}\frac{U}{2}-1, & U<U_{c}=-1, \\ \frac{U}{2}+1, & U>\widetilde{U}_{c}=1 .\end{cases}
$$

This result entails that the spectrum is turned upside down when changing the interaction from attractive to repulsive, the critical points being symmetric with respect to the free case, $U=0$. The same analysis is not that clean for the system with a molecule. The precise values for $\widetilde{U}_{c}$ and $E_{c}(U)$ depend on the molecular parameters $\omega$ and $g$. For $\omega \neq 0$ and $g \neq 0$ the symmetry around $U=0$ is broken. Furthermore, due to the smaller values of the total number of particles $N$ accessible to our current computational power, it has not been possible to obtain a precise estimate of the behavior in the thermodynamic limit. For $g=\omega=5$ a numerical fit to the case with $N=320$

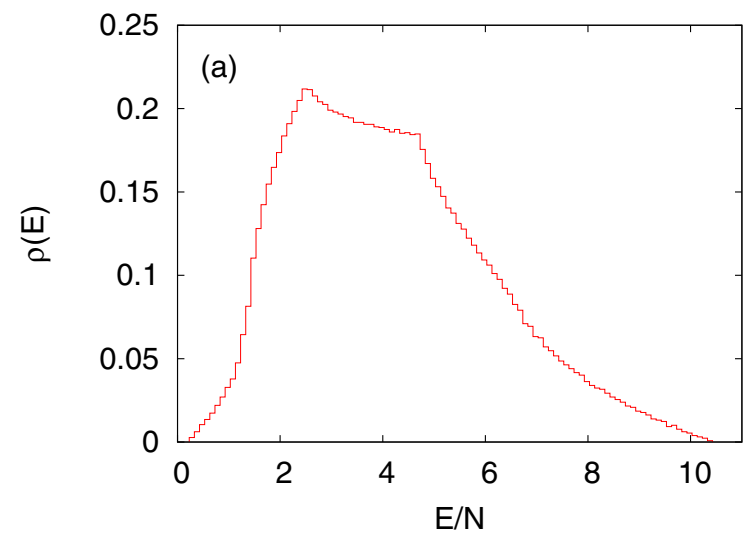

particles gives rise to

$$
E_{c}= \begin{cases}0.46 U-1.89, & U<U_{c} \sim-1.3, \\ 0.42 U+2.55, & U>\widetilde{U}_{c} \sim 1.7,\end{cases}
$$

where both critical values, $U_{c}$ and $\widetilde{U}_{c}$, correspond to the finitesize precursors, which are still far from the true critical points in the thermodynamic limit.

It is possible to study ESQPT for fixed values of the system parameters by looking at the density of states $\rho(E)$ as a function of the excitation energy. A singular behavior in $\rho(E)$ signals an ESQPT at the critical energy $E_{c}$. It has been recently shown that $\rho(E)$ presents a logarithmic singularity at $E=E_{c}$ for models with just one semiclassical degree of freedom. On the contrary, the density of states for models with more than one semiclassical degree of freedom is not singular. In these cases, the logarithmic singularity occurs in its derivative $\rho^{\prime}(E)$ [37]. This feature has been tested in the integrable Tavis-Cummings model with one semiclassical degree of freedom, and in the nonintegrable Dicke model with two semiclassical degrees of freedom [17]. Following a similar procedure, we fix the value of the interaction $U=9$ and calculate the density of states as a function of the excitation

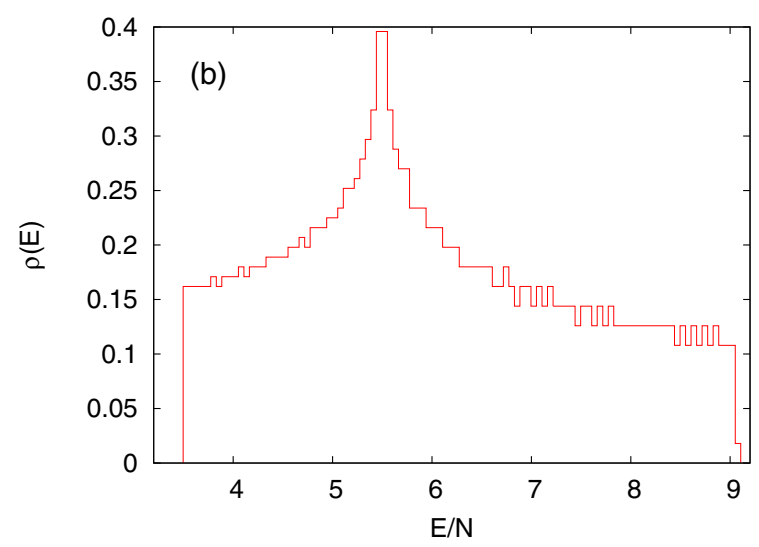

FIG. 7. (Color online) Density of states $\rho(E)$ : Panel (a) for $N=320, U=9$ and a molecule at resonance. Panel (b) for $N=2000$ without molecule. 

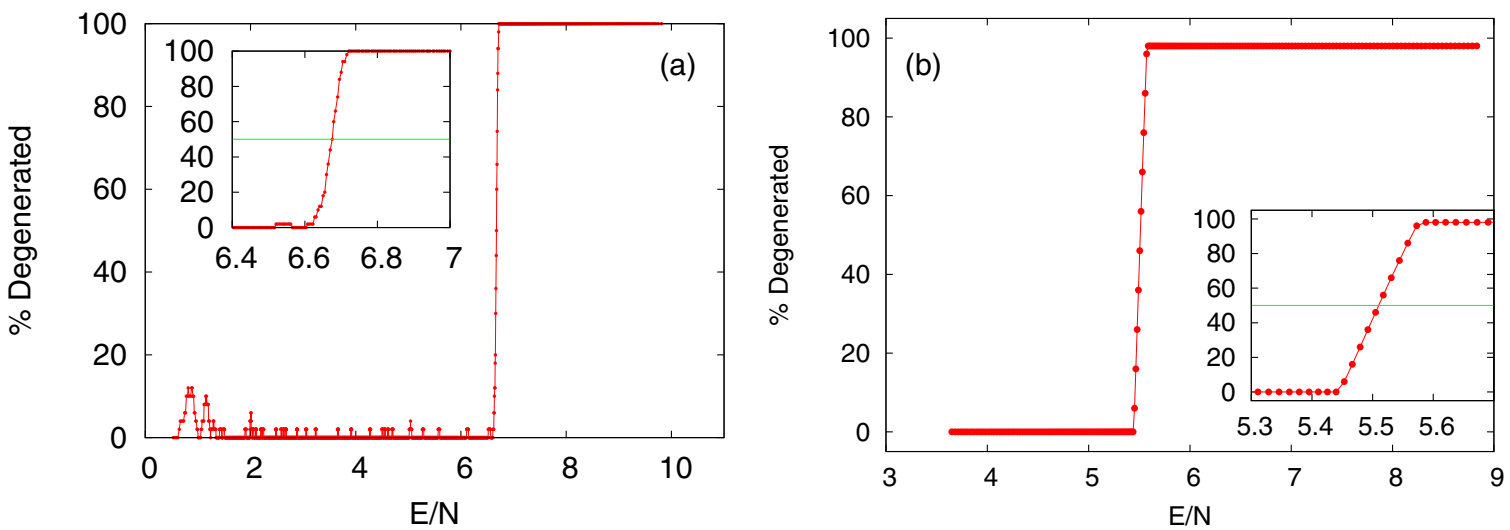

FIG. 8. (Color online) Ratio of degenerate energy levels as a function of the energy: panel (a) for $N=320, U=9$ and a molecule at resonance. Panel (b) for $N=2000$ without molecule.

energy for the two systems. From the results of Eqs. (35) and (36) we estimate the critical energy to be around $E \sim 6.3$ in the case with molecule, and $E \sim 5.5$ in the case without molecule.

Figure 7 shows the density of states $\rho(E)$ versus the excitation energy. Panel (a) corresponds to the case of a molecule at resonance and $N=320$ particles. It shows no neat trace of a singular behavior around the estimated critical energy $E_{c} \sim 6.3$. This is however compatible with a logarithmic singularity in the derivative of the density of states, which is very difficult to see in systems of this size. Panel (b) corresponds to $N=2000$ without molecule. It clearly shows a precursor of the logarithmic singularity at $E_{c}=5.5$. The results obtained with these two systems are therefore, compatible with those derived in Ref. [17] for the Tavis-Cummings and Dicke models.

In order to get a deeper insight into the properties of the ESQPTs, we plot in Fig. 8 the ratio of degenerate energy levels as a function of the scaled energy $E / N$. The calculation has been done in the following way: (a) we choose a set of 100 energy levels, starting from the ground state; (b) we count the number of pairs whose relative energy difference is less than $10^{-6}$; (c) we normalize this value to 100 ; (d) we associate this value to an energy equal to the mean energy of the interval; (e) we repeat the calculation starting from the 11th energy level; (f) we proceed in the same way up to covering the whole spectrum.
In the same figure we plot the results for the system with (a) and without the molecule (b). The case without molecule shows an abrupt transition from nondegenerate to degenerate energy levels at the expected value for the critical energy $E_{c} \sim 5.5$. The case with molecule is not as sharp, though the critical behavior is still clearly observed. For this case, a small number of degeneracies appear distributed randomly in the lower part of the spectrum. We attribute this behavior to the spectral fluctuations of the energy levels, and relate it to the crossings and avoided crossings shown in Fig. 5 in the symmetric phase. In spite of this behavior at low energies, we observe an abrupt change from nondegenerate to degenerate in the spectrum at an energy $E_{c} \sim 6.6$. The 5\% discrepancy with our estimate is attributed to finite-size effects.

Figure 9 shows the behavior of the order parameter $\sqrt{F_{Q F I}}$ for both systems, with molecule (a) and without molecule (b). It has been obtained by calculating the square root of the Fisher information for all the eigenstates of the systems. The results are similar to those obtained for the density of states. For the case without the molecule the quantum Fisher information displays a sharp singularity at the estimated critical energy $E_{c} \sim 5.5$. On the contrary, there is no such a signature in the case with molecule. These results are compatible with the fact that ESQPTs are softer in systems with more than one semiclassical degree of freedom, as it is pointed out in [16,37].
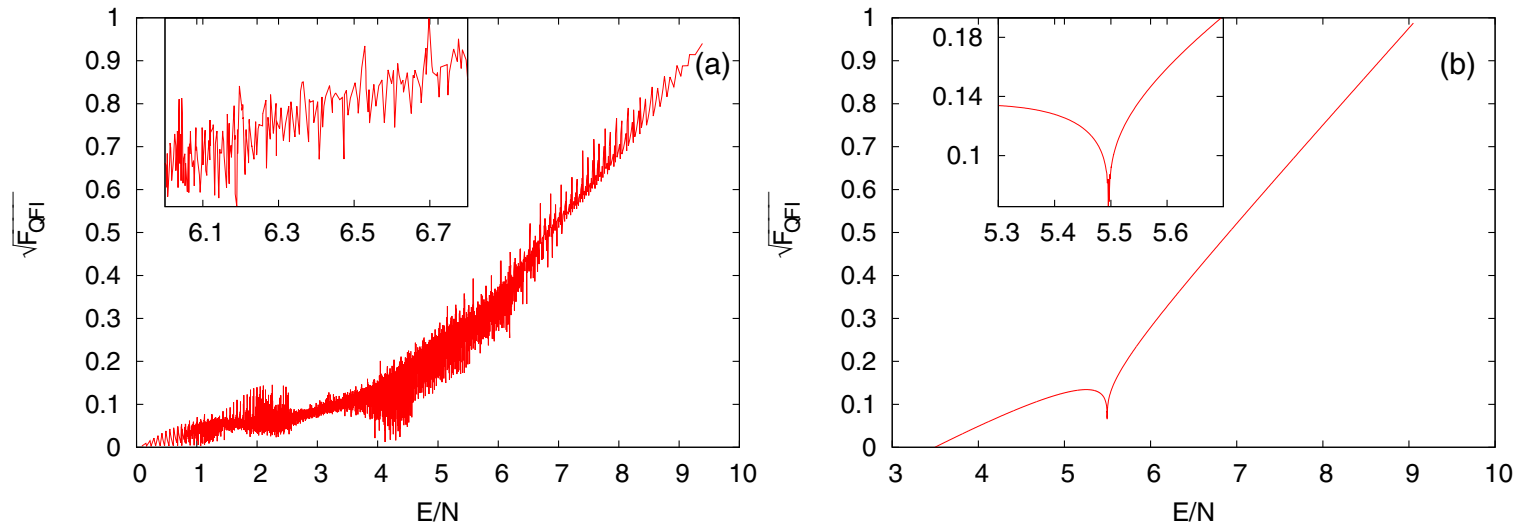

FIG. 9. (Color online) Fisher information as a function of the energy: Panel (a) for $N=320, U=9$ and a molecule at resonance. Panel (b) for $N=2000$ without molecule. 


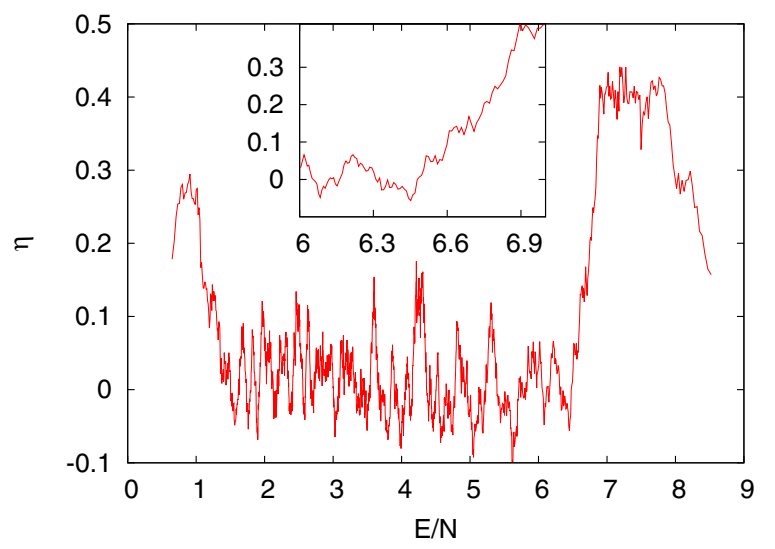

FIG. 10. (Color online) Spectral statistics for $U=9$ and $N=$ 360 , even-parity states, as a function of the scaled energy $E / N$.

In the case with the molecule, large fluctuations between different eigenstates blur the expected singular behavior of the order parameter at the estimated critical energy. On the other hand, in the limit without molecule, which has just one semiclassical degree of freedom, a cusp singularity at the critical energy is clearly seen.

Finally, it is interesting to analyze the degree of chaos in the spectra as a function of the energy. In Ref. [10] was conjectured a connection between the ESQPT and the onset of chaos in the Dicke model. Here we analyze the nearest-neighbor spacing distribution as the simplest measure of quantum chaos. While integrable systems follow a Poisson distribution $P_{P}(s)=\exp (-s)$, nonintegrable and fully chaotic systems are well described by the Wigner surmise $P_{W}(s)=$ $\frac{\pi}{2} s \exp \left(-\frac{\pi}{4} s^{2}\right)$. Given a set of spacings $s_{i}=\left(E_{i+1}-E_{i}\right) /\langle s\rangle$, a natural way to measure the degree of chaos is by means of the ratio

$$
\eta=\frac{\operatorname{var}_{s}-\operatorname{var}_{W}}{\operatorname{var}_{P}-\operatorname{var}_{W}}
$$

where "var" means the variance of the distribution. The parameter $\eta$ varies from $\eta=0$ for fully chaotic systems, to $\eta=1$, for integrable systems.

In Fig. 10 we show the parameter $\eta$ as a function of the scaled energy $E / N$. The calculation has been done as follows: (a) a set of 250 levels, starting from the ground state has been chosen, (b) the set has been unfolded, discarding a $20 \%$ of higher and lower levels, (c) the parameter $\eta$ has been calculated, (d) the value of $\eta$ has been linked to the mean energy value of the set, (e) the procedure has been repeated increasing the energy. It is important to note that all the eigenstates have a definite value of parity. As can be seen in Fig. 10, a transition from a fully chaotic to an intermediate regime takes place at $E \sim E_{c}$. Below this critical point, the system is more or less chaotic, with the exception of the region very close to the ground state, which is closer to integrability. This is fully compatible with the complex behavior shown in Fig. 5 for the lowest excited states in the symmetric phase. On the contrary, above the critical energy the system is far from chaos. The interplay between chaos and regularity as a function of energy is qualitatively similar to the Dicke model $[10,38]$. For Dicke and the present model, chaos characterizes the normal phase, in which there are no degeneracies, whereas the symmetry-breaking phase is closer to integrability. Despite this fact, there seems to be no clear connection between the ESQPT and the onset of chaos [38]. Dynamics of systems with two semiclassical degrees of freedom suffering ESQPTs seems to be more chaotic in the phase in which there are no degeneracies in the spectrum.

\section{CONCLUSIONS}

By means of analytic and numerical results, we have studied the QPT signaled by the population imbalance in two kinds of double-well BECs: one comprising bosonic atoms tunneling between the two wells, and the second including the interaction of the atoms with a diatomic molecule. Our main results are summarized as follows:

Both systems display a second-order QPT at a certain critical value $U_{c}$ of the atom-atom interaction. In both cases, for $U<U_{c}$ the ground state is degenerate due to the breaking of the left-right symmetry in the atomic system, giving rise to a finite population imbalance. On the contrary, for $U>U_{c}$ the ground state is not degenerate and the imbalance is zero. This critical value of the control parameter was obtained by means of a mean-field calculation, and it was numerically tested by means of exact diagonalizations and finite-size scaling using the gap between the ground state and the first excited state as a signature of the transition. We have also related the population imbalance with the square root of the Fisher information, and tested the mean-field with exact diagonalizations.

Beyond mean-field techniques that take into account the quantum fluctuations have been used to describe the low energy spectrum. A comparison with exact diagonalizations shows the goodness of these theoretical results. In particular, it is worth mentioning that the theory successfully accounts for the crossings between levels corresponding to opposite parities, which take place when the degree of freedom of the diatomic molecule is added. This formalism greatly improves the description of the critical point, showing that the second-order QPT is strongly influenced by correlations and fluctuations that are beyond the mean-field approximation.

Both systems also display ESQPTs at certain critical energies depending on the value of the interaction $U$. Contrary to the ground state QPT, the nature of these transitions differs in both models. The two-site Bose-Hubbard Hamiltonian is characterized by a $\lambda$ divergence in the density of states. This behavior in the energy spectrum is comparable to the Lipkin and the Tavis-Cummings models, both having a single semiclassical degree of freedom. On the contrary, the density of states for the case in which the atoms interact with a diatomic molecule shows no divergence. This behavior is qualitatively similar to the Dicke model, in which the divergence arises in the derivative of the density of states.

Finally, we can infer a correlation between the ESQPT and the onset of chaos in the systems with atoms and molecules. A similar conclusion has been obtained for the Dicke model [10], though it seems that the transition between the integrable and the chaotic regime does not coincide with 
the critical energy at which the ESQPT takes place [38]. Taking into account the strong numerical limitations for the atom-molecule system, we conjecture that quantum chaos only appears in the nondegenerate region of the spectrum. Whether this is a generic mechanism, linking chaos and critical phenomena in the energy spectrum of systems with more than one semiclassical degree of freedom remains still an open question.

\section{ACKNOWLEDGMENTS}

This work is has been partially supported by the Spanish MINECO Grants No. FIS2012-35316, No. FIS2012-34479, and No. FIS2011-28738-c02-01, by Junta de Andalucía under group number FQM-160 and Project P11-FQM-7632, and by the Consolider-Ingenio 2010 Programme CPAN (CSD200700042).
[1] M. Albiez, R. Gati, J. Fölling, S. Hunsmann, M. Cristiani, and M. K. Oberthaler, Phys. Rev. Lett. 95, 010402 (2005).

[2] G. S. Paraoanu, S. Kohler, F. Sols, and A. J. Leggett, J. Phys. B 34, 4689 (2001).

[3] A. P. Tonel, J. Links, and A. Foerster, J. Phys. A 38, 1235 (2005).

[4] B. Juliá-Díaz, D. Dagnino, M. Lewenstein, J. Martorell, and A. Polls, Phys. Rev. A 81, 023615 (2010).

[5] V. S. Shchesnovich and V. V. Konotop, Phys. Rev. Lett. 102, 055702 (2009).

[6] T. Zibold, E. Nicklas, C. Gross, and M. K. Oberthaler, Phys. Rev. Lett. 105, 204101 (2010).

[7] M. A. Caprio, P. Cejnar, and F. Iachello, Ann. Phys. (N.Y.) 323, 1106 (2008).

[8] S. Sachdev, Quantum Phase Transitions (Cambridge University Press, Cambridge, England, 1999).

[9] A. Relaño, J. M. Arias, J. Dukelsky, J. E. García-Ramos, and P. Pérez-Fernández, Phys. Rev. A 78, 060102 (2008); P. PérezFernández, A. Relaño, J. M. Arias, J. Dukelsky, and J. E. GarcíaRamos, ibid. 80, 032111 (2009).

[10] P. Pérez-Fernández, A. Relaño, J. M. Arias, P. Cejnar, J. Dukelsky, and J. E. García-Ramos, Phys. Rev. E 83, 046208 (2011).

[11] R. Puebla, A. Relaño, and J. Retamosa, Phys. Rev. A 87, 023819 (2013); R. Puebla and A. Relaño, Europhys. Lett. 104, 50007 (2013).

[12] J. M. Arias, J. Dukelsky, and J. E. García-Ramos, Phys. Rev. Lett. 91, 162502 (2003).

[13] P. Cejnar, J. Jolie, and R. F. Casten, Rev. Mod. Phys. 82, 2155 (2010).

[14] P. Ribeiro, J. Vidal, and R. Mosseri, Phys. Rev. E 78, 021106 (2008).

[15] F. Pérez-Bernal and O. Álvarez-Bajo, Phys. Rev. A 81, 050101(R) (2010).

[16] P. Pérez-Fernández, P. Cejnar, J. M. Arias, J. Dukelsky, J. E. García-Ramos, and A. Relaño, Phys. Rev. A 83, 033802 (2011).

[17] T. Brandes, Phys. Rev. E 88, 032133 (2013).
[18] V. M. Bastidas, P. Pérez-Fernández, M. Vogl, and T. Brandes, Phys. Rev. Lett. 112, 140408 (2014).

[19] B. Dietz, F. Iachello, M. Miski-Oglu, N. Pietralla, A. Richter, L. von Smekal, and J. Wambach, Phys. Rev. B 88, 104101 (2013).

[20] M. A. Alcalde, M. Bucher, C. Emary, and T. Brandes, Phys. Rev. E 86, 012101 (2012).

[21] E. Timmermans, P. Tommasini, R. Côté, M. Hussein, and A. Kerman, Phys. Rev. Lett. 83, 2691 (1999).

[22] G.-R. Jin, C. K. Kim, and K. Nahm, Phys. Rev. A 72, 045602 (2005).

[23] G. Santos, A. Tonel, A. Foerster, and J. Links, Phys. Rev. A 73, 023609 (2006).

[24] A. Motohashi and T. Nikuni, Phys. Rev. A 82, 033631 (2010).

[25] J. C. Sanders, O. Odong, J. Javanainen, and M. Mackie, Phys. Rev. A 83, 031607(R) (2011).

[26] G. J. Milburn, J. Corney, E. M. Wright, and D. F. Walls, Phys. Rev. A 55, 4318 (1997).

[27] A. J. Leggett, Rev. Mod. Phys. 73, 307 (2001).

[28] M. Holthaus and S. Stenholm, Eur. Phys. J. B 20, 451 (2001).

[29] M. Chuchem, K. Smith-Mannschott, M. Hiller, T. Kottos, A. Vardi, and D. Cohen, Phys. Rev. A 82, 053617 (2010).

[30] M. Mel-Messeguer, B. Juliá-Díaz, and A. Polls, J. Low Temp. Phys. 165, 180 (2011).

[31] J. Dukelsky, C. Esebbag, and P. Schuck, Phys. Rev. Lett. 87, 066403 (2001).

[32] H.-Q. Zhou, J. Links, R. H. McKenzie, and X.-W. Guan, J. Phys. A 36, L113 (2003).

[33] G. Ortiz, R. Somma, J. Dukelsky, and S. Rombouts, Nucl. Phys. B 707, 421 (2005).

[34] G. Mazzarella, L. Salasnich, A. Parola, and F. Toigo, Phys. Rev. A 83, 053607 (2011).

[35] R. Gilmore and D. H. Feng, Nucl. Phys. A 301, 189 (1978).

[36] J. P. Blaizot and G. Ripka, Quantum Theory of Finite Systems (MIT Press, Cambridge, MA, 1986).

[37] P. Stránský, M. Macek, and P. Cejnar, Ann. Phys. 345, 73 (2014)

[38] M. A. Bastarrachea-Magnani, S. Lerma-Hernández, and J. G. Hirsch, Phys. Rev. A 89, 032102 (2014). 